\title{
Coherent motion of dense active matter
}

\author{
Amin Doostmohammadi and Julia M. Yeomans ${ }^{\mathrm{a}}$
}

The Rudolf Peierls Centre for Theoretical Physics, University of Oxford, 1 Keble Road, Oxford OX1 3NP, UK

Received 6 November 2017 / Received in final form 22 December 2017 Published online 28 March 2019

\begin{abstract}
We discuss experiments, simulations and theories showing how active nematics behave in circular and linear confinement, and in the presence of friction. In each case active turbulence can be suppressed resulting in steady or periodic flows. These have the potential to act as power sources, transforming chemical energy to mechanical work, and we review first steps in this direction.
\end{abstract}

\section{Introduction}

Active matter takes energy from its surroundings on a single particle level and uses it to do work, often in the form of movement. Studying active materials has become an increasingly popular field of research in the last few years for several reasons. Firstly, active matter systems are out of thermodynamic equilibrium, and provide examples of non-equilibrium statistical physics. Indeed the collective behaviour of active matter is very rich, ranging from wound healing in cell layers to the evolving patterns formed by flocks of starlings in flight. Secondly, nature has designed very efficient biological machines and the ability to emulate them will lead to novel nanotechnologies. Thirdly, biological systems are usually active, and the ideas of active matter are suggesting new ways of thinking about biological processes. Reviews of active matter can be found in $[1-3]$.

Any living creature can be thought of as an active system. However it is easiest to understand simpler organisms and the majority of research has been devoted to cells and molecular motors, but with interesting exceptions such as fire ants and starlings. Here we shall focus on dense active matter, where hydrodynamic interactions are at least to some extent important, and our experimental examples will be swimming bacteria [4], confluent cell layers [5] and suspensions of microtubules driven by twoheaded kinesin motors [6]. It is also possible to fabricate active particles, typically colloids driven by phoretic forces or external stimuli such as light or electric fields [7]. The majority of experiments so far are on two-dimensional systems, where imaging is much easier.

Some active particles, such as driven microtubules or neural progenitor stem cells, which continuously reverse their direction of motion have nematic (head-tail) symmetry. Others, such as motile bacteria have a head-tail asymmetry and thus a clear polarity. In both cases experiments have shown that nematic, rather than polar,

a e-mail: julia.yeomans@physics.ox.ac.uk 

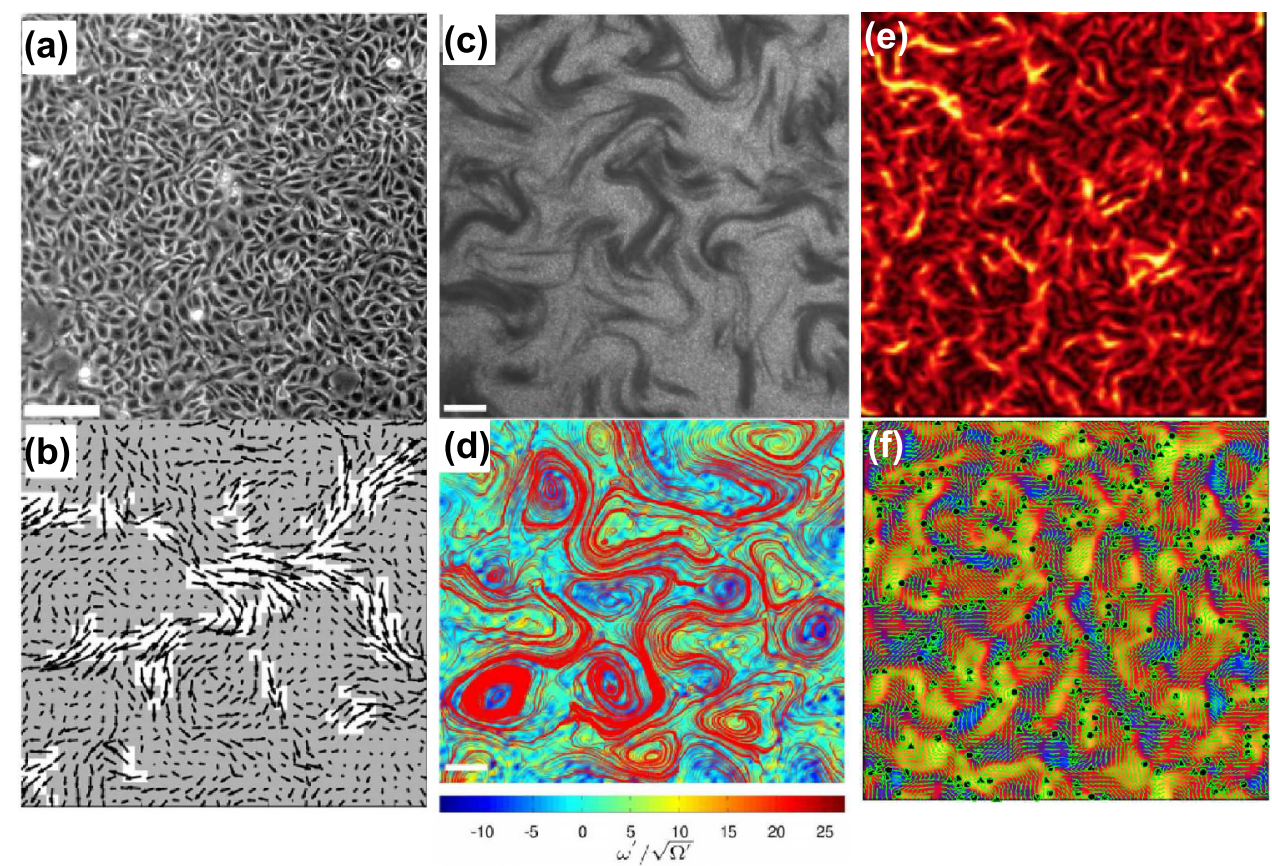

Fig. 1. Active turbulence in biological fluids. Shown here, are seemingly-chaotic flow patterns for (a), (b) MDCK cells [8], (c), (d) swarms of sperm [9], and (e), (f) active nematic simulations [10]. The vectors in (b) show the velocity field and colourmaps in (d) illustrate the vorticity field. In (e) and (f) instantaneous contours of velocity magnitude and vorticity are shown, respectively. Green lines are the nematic director field while symbols represent $+1 / 2$ (circles) and $-1 / 2$ (triangles) topological defects.

defects appear in dense systems $[11,12]$ but the reasons for this are not fully understood. However, both polar and nematic active particles that are self-propelled with no external forces produce stresslet far flow fields with nematic symmetry. This gives rise to a term $\zeta \mathbf{Q}$ in the stress tensor in the continuum equations of motion, where $\zeta$ measures the strength of the activity and $\mathbf{Q}$ is the nematic order parameter [13].

Noting that the active term appears in the stress tensor, under a derivative, any gradient in the direction or magnitude of the nematic order induces a stress, and hence a flow. In turn the flow destabilises the nematic order. The instabilities in the director field localise to form lines of high elastic distortions, which we will call 'walls'. At the walls, the elastic distortions are released by nucleating pairs of $\pm 1 / 2$ topological defects. The $+1 / 2$ defect is motile, because its asymmetric director field implies unbalanced stresses, and hence it can escape from the $-1 / 2$ defect before they annihilate. It moves away until it meets another $-1 / 2$ defect and only then is destroyed. Hence the steady state of active turbulence corresponds to a finite defect density, which should be contrasted to passive nematics where, in the absence of pinning, defects eventually anneal out.

The distortions in the director field give rise to flow jets and a velocity field that is characterised by high vorticity (Fig. 1). This dynamical state is termed active or mesoscale turbulence, and is described in more detail in the Geilo notes from the 2015 school ([14] and references therein). Note that mesoscale turbulence differs from inertial turbulence: any momentum due to velocity fluctuations is immediately damped by high viscosity since the mesoscale turbulence essentially occurs at zero Reynolds number. Moreover in mesoscale turbulence energy is pumped in at microscopic length 
scales, and dissipated at the vortex length scale, whereas in inertial turbulence it cascades from macroscopic scales down to the Kolmogorov length.

A characteristic feature of dense active nematics is the existence of a steady state population of $\pm 1 / 2$ topological defects. The activity creates pairs of defects, helped by the pre-existing director deformation at the walls in the director field. The $+1 / 2$ defect is motile, because its asymmetric director field implies unbalanced stresses, and hence it can escape from the $-1 / 2$ defect before they annihilate. It moves away until it meets another $-1 / 2$ defect and only then is destroyed. Hence the steady state of active turbulence corresponds to a finite defect density, which should be contrasted to passive nematics, where in the absence of pinning, defects eventually anneal out.

The disordered nature of active turbulence means that it is likely to be difficult to harness its energy. Hence it is interesting to consider ways to 'tame' active turbulence, channelling the energy input into coherent flows. In these notes we summarise recent research showing how this can be done by screening hydrodynamic effects through confinement, or by friction. Finally, we discuss possible ways of applying the understanding gained from active flows in confinement to designing active microfluidics and active micromachines in the light of recent experimental and computational advances in these directions.

\section{Active flow in confinement}

We begin by reviewing experimental, theoretical, and numerical studies of the effects of confinement on the dynamical behaviour of active matter. The basic role of a confinement is to introduce a length scale in addition to the intrinsic length scale of the flow produced by the active matter. Two types of geometrical constraints are commonly used to confine active systems: circular confinements as isotropic constraints, while rectangular channels induce anisotropy in the flow. In what follows, we will discuss how these two geometries lead to distinct and surprisingly complex patterns of motion.

\subsection{Circular confinement}

Circular confinement of active matter results in steady, or periodic, vortical flow fields, that reflect the confining geometry. However, details of the flow are strongly system-dependent, affected by surface interactions, steric inter-particle forces and hydrodynamic interactions between the active particles. This is well demonstrated in experiments on a dense suspension of Bacillus subtilis in a flattened drop of diameter $\sim 50 \mu \mathrm{m}$ which organise to drive a spiral vortical flow (Fig. 2a) [15]. A boundary layer of cells, which line up at an angle to the edge of the drop, move in a well-defined direction around the boundary, and produce strong flows that drive the fluid in the bulk of the drop in the opposite direction. The cells in the middle of the drop swim against this flow, but their net motion is backwards. To help sort out details of the motion the experiments were compared to simulations of swimmers modelled as ellipsoids interacting through steric forces and torques [16]. Each particle produced an extensile stresslet flow field (pulling fluid towards its sides and pushing it outwards along its length), and the stress-free boundary was introduced into the simulations using the method of images. The simulations showed that the most important factor determining the flow patterns is the interaction of the swimmers with their collectively generated fluid flow.

In an extension to more complex geometries Wioland et al. [17] considered lattices of circular cavities each confining a bacterial vortex and connected by short 

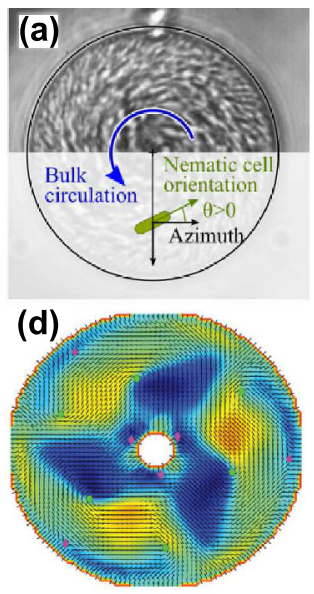
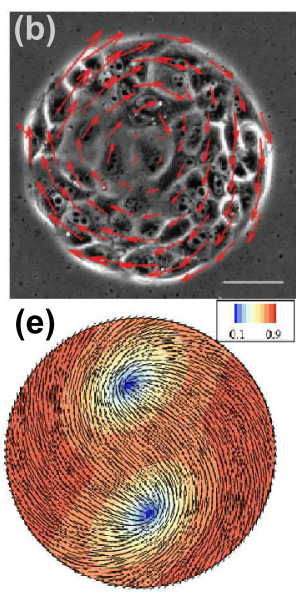
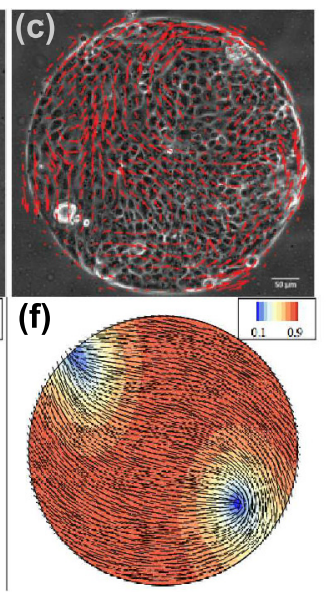
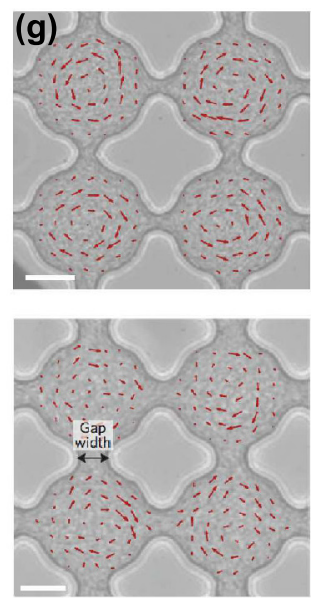

Fig. 2. Flow and topological defect patterns in circular confinements. (a) Circulation of confined B. subtilis bacterial suspension [15]. (b) Uniform circulation and (c) active turbulence of MDCK cells within different circle sizes [19]. (d) Active nematic simulations of flow within an annulus [20]. The colourmap represents the vorticity field. Circles (green) and diamonds (magenta) mark $+1 / 2$ and $-1 / 2$ topological defects, respectively. (e), (f) Time evolution of director patterns and defect trajectories in simulations of active nematics confined in a circle [18]. (g) Counter-rotating and co-rotating circulations of B. subtilis bacteria within interconnected circular confinements [17].

channels (Fig. 2g). Continuity of the edge currents of bacteria meant that the lattice flow self-organised. For wider channels between neighbouring chambers, the boundary bacterial flows stream between the cells giving rise to a checkerboard pattern of clockwise and anti-clockwise vortices. For smaller channels, they are confined to their own cavities and the hydrodynamic coupling between chambers tends to stabilise rotation in the same direction in each cell. A similar crossover between intra- and inter-chamber flow has recently been interpreted as analogous to a Fredericks' transition (which describes the competition between boundary and field-induced ordering in a passive nematic liquid crystal) [18].

Continuum theories of active nematics also demonstrate the possibility of stabilising active turbulence in circular or cylindrical confinement [18,20-25]. These calculations emphasise the role of topological defects in controlling the flow. Circular confinement with planar or homeotropic boundary conditions introduces a +1 topological defect. This splits into two $+1 / 2$ defects which act as local pumps for the flow because the surrounding distortions in the director field induce active stresses such that each $+1 / 2$ defect becomes self-motile. The defects are driven towards opposing walls where they can remain pinned and stabilise arrays of flow vortices (Fig. 2d), or continue to move, along periodic closed orbits. They can also be absorbed by the walls and then recreated in a manner similar to the production of defects in active turbulence, giving rise to circular flows that periodically oscillate in strength (Figs. 2e and $2 \mathrm{f}$ ). Periodic flows of four defects have also been reported [18].

At larger confinement sizes, active turbulence is restored where $-1 / 2$ defects interact elastically with the walls and are pushed close to the boundaries, while $+1 / 2$ defects navigate through the bulk creating irregular flows. It is worth noting that the emergence of circulating and turbulent flows depending on the confinement size have been also shown using mean-field kinetic theories [26]. There, the defect dynamics are not taken into account and thus ordered-dynamic states are not considered. Nevertheless, the results conform with the general picture that the circular collective 
motion spanning the whole domain is achieved once the confinement size becomes commensurate with the intrinsic vorticity length-scale that is set by the activity of the fluid. Above this critical domain size, the system moves towards the active turbulence state.

Coherent flows have also been observed in MDCK cells confined to a circular adherent domain surrounding by a cell-repellent coating $[19,27,28]$. Once the cells had divided to form a dense 2D circular layer coherent rotational motion (Fig. 2b), with occasional stochastic reversals were observed. This was observed only in the smaller circles $(100$ and $200 \mu \mathrm{m})$, not in larger confinement, where bursts of activity were more reminiscent of active turbulence (Fig. 2c). The circulations were destroyed by knocking out cell-cell junctions, emphasising the importance of collective interactions in driving the coherent flow patterns. Cell division has also been implicated in driving rotation of cell clusters [29,30]. The cells also performed regular breathing oscillations in the radial direction. The source of such breathing oscillations are not fully understood but may arise from coupling to signaling molecules.

\subsection{Active flow in a channel}

\subsubsection{Theories and simulations}

Figure 3 summarises the flow patterns which result when an active nematic is confined to an infinitely long channel [20]. These results were obtained by numerically solving the continuum equations of active nematics.

At low activity for uniform nematic ordering there is no flow. At a threshold value of the activity which depends on the size of the system, the director field spontaneously transforms to a non-uniform configuration that drives laminar flow. This was first predicted by Voituriez et al. [31] using a mathematical analogy to the Fredericks? transition of a nematic liquid crystal in a magnetic field, although they predicted the emergence of shear flow rather than unidirectional flow due to a no-net-flow constraint in their analysis. Numerical studies on quasi-one-dimensional setups have confirmed this behaviour for confined active systems and showed that the emergence of spontaneous laminar flow depends on three main factors: the type of the activity (extensile vs contractile), the response of elongated active components to the gradients in the flow (flow aligning vs flow tumbling), and the preferred orientation of the active particles at the walls of the channel $[32,33]$. We note that the activity threshold goes to zero in the absence of confining walls, as noted in an earlier work by Simha and Ramaswamy [34] who showed that without confinement dense extensile (contractile) active suspensions are always unstable to bend (splay) instabilities. Moreover there are certain non-uniform director configurations, resulting from surface curvature or boundary anchoring, that can result in flow for arbitrarily small activity even in confined geometries [35].

Increasing the activity, the correlation length associated with the vorticity decreases. The streamlines first become oscillatory and then, as the vortex correlation length becomes of order the channel height, stabilise to a one-dimensional line of opposing vortex rolls (Fig. 3d). Similar behaviour has been observed for contractile polar fluids modeling actomyosin cortex flow within free-slip boundaries representing cytosol and membrane [37] and also in kinetic mean-field simulations [26]. However, rather more surprisingly, here the vortices are accompanied by a dynamically ordered array of topological defects. The $+1 / 2$ defects move past each other on sinusoidal trajectories, with half moving to the right, and half to the left. They transiently form pairs, in a way reminiscent of the great chain figure in country dancing - hence we have termed this state the ceilidh dance. The defect movement leads to periodic 


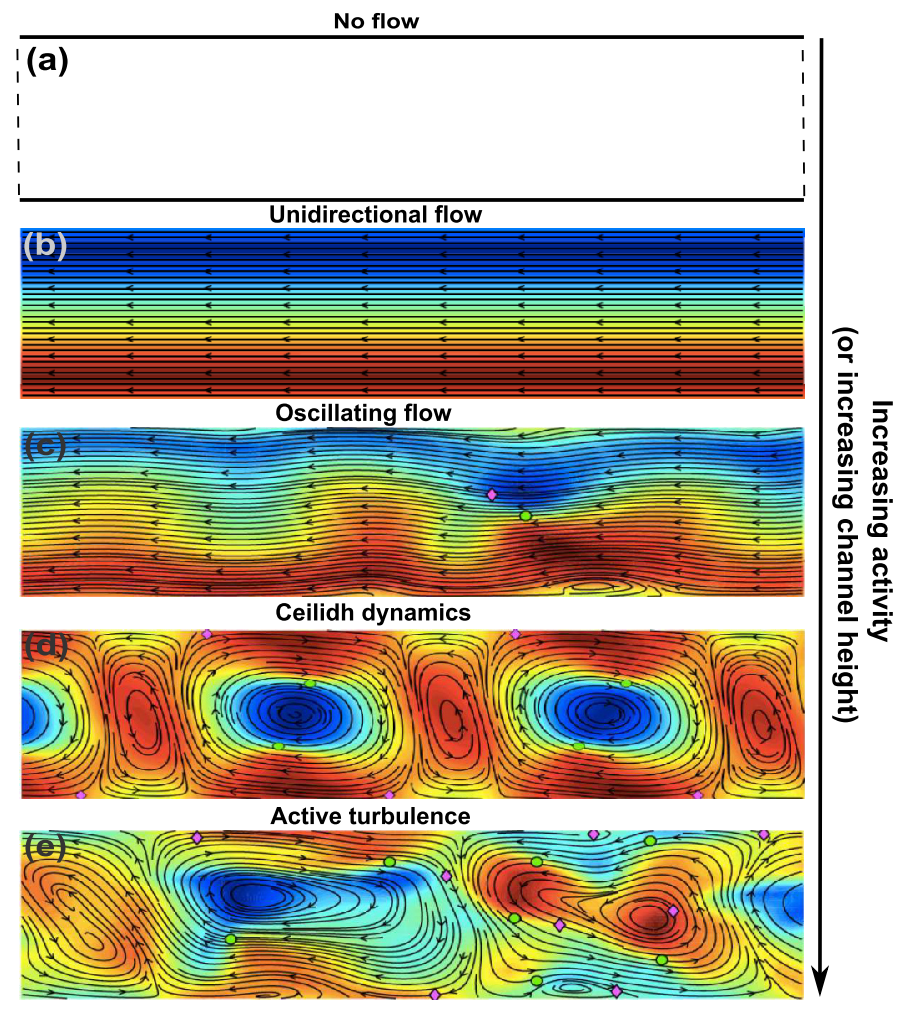

Fig. 3. Distinct flow states of active nematics confined in a channel. The black lines represent the streamlines of the velocity field and the black arrows indicate the direction of the flow. The colourmap represents the vorticity field. Circles (green) and diamonds (magenta) mark $+1 / 2$ and $-1 / 2$ topological defects, respectively $[20,36]$.

changes in the shapes of the vortices. Meanwhile, the $-1 / 2$ defects are localised near the walls of the channel, where they oscillate weakly about their mean position.

A further increase in activity decreases the vortex size, locally destabilising the ceilidh dance to form patches of active turbulence, which can be identified by measuring their enstrophy (relative to that of the background vortex lattice) [36]. The turbulent patches continually appear, combine and die out, giving a kymograph (space-time diagram) reminiscent of directed percolation. Measuring the exponents associated with the transition where the turbulent cluster first spans the system indicates that this transition is indeed in the directed percolation universality class. The same critical behaviour is associated with the transition to inertial turbulence in channel flow $[38,39]$. However, in the inertial case the turbulent patches must be injected, here they occur due to intrinsic active fluctuations.

Simulations have also been used to study viscoelastic active nematic flows in channel confinement, showing that further complexity introduced by the viscoelastic time scale can lead to additional states such as shuffling flows where the whole system oscillates along the flowing direction [40].

\subsubsection{Experiments}

Wioland et al. [41] have performed experiments on dense suspensions of Bacillus subtilis moving around a racetrack, with sufficiently long straight sections that it 
is reasonable to assume that the behaviour mimics that in a channel. They see a crossover from laminar flow to active turbulence as the channel height is increased, when the vortices formed by the bacteria are of a size of order the channel height. Near the crossover they report states where the flow oscillates, and where it forms vortices which look similar to the patterns in Figure 3d. However, as in their experiments in circular confinement, the interactions of individual swimmers with the channel boundaries are identified as an important factor in controlling the bulk flow configuration.

Experiments on 2D confluent cell layers have concentrated on a different protocol [42-45]. It is an extra experimental step to stop cells dividing, so a much more natural experiment than confining cells in channels is to consider cell layers expanding along channels, in this case provided by fibronectin stripes surrounded by nonadhesive areas. It is apparent that the collective behaviour of the cells is key in determining how the channels fill, but there is no complete understanding of either the physical or biological mechanisms that underlie this. The cells appear to move faster in narrower channels, less than a few cell diameters, where they are more aligned along the flow direction, and a pulsatile flow has been observed. Cell division events also appear to be predominantly aligned with the channel length. In wider channels vortices are seen, but there is no evidence that these form a regular pattern. This behaviour has some similarities to that of the continuum models, but more work needs to be done to understand effects such as compressibility and cell proliferation. Other explanations, such as the existence of leader cells [46] and diffusion-drift models [43,44], have also been considered.

Finally, an interesting example of confined active flow is cytoplasmic streaming in plant cells [47]. Myosin coated organelles move along actin filament bundles fixed at the edge of the cell. This entrains cytoplasm, driving flows at $1-100 \mu \mathrm{m} \mathrm{s}^{-1}$ which aids transport of nutrients and organelles around the cell. The mechanism can be distinguished from true 'active flow' as it is driven by effective pumps at the cell membrane. However it shares the features of organised active fluid transport in confinement.

\section{Friction}

Most experiments on active nematics are in two dimensions, and deal with active particles on a substrate. Therefore substrate friction will be an important factor in determining the dynamical behaviour. We note that there is a clear distinction between frictional damping and viscous dissipation. Viscous dissipation is an internal mechanism to the dynamics of the active fluid and therefore does not affect the momentum conservation. On the other hand, friction provides a means of external damping of the momentum, leading to hydrodynamic screening of the flow. The friction between active particles and their environment is indeed present in many 'wet' systems such as bacteria that are contacting a surface, or cells crawling on a substrate. Continuum simulations have shown that, as friction is increased, active turbulence is still observed, but with a decrease in the mean velocity and an increase in the number of topological defects $[48,50]$. This prediction has now been confirmed experimentally in microtubule-motor systems. The two dimensional active layer was formed at a surfactant-decorated oil-water interface, and friction was varied by changing the viscosity of the oil over several orders of magnitude [51] (We refer to this as frictional damping as momentum is dissipated external to the active fluid, in the oil).

Friction screens the hydrodynamics to a length scale $\sqrt{\nu / \gamma}$ where $\nu$ is the kinematic viscosity and $\gamma$ a measure of the strength of the friction. Therefore one might expect a qualitative change in behaviour when this screening length becomes of order 


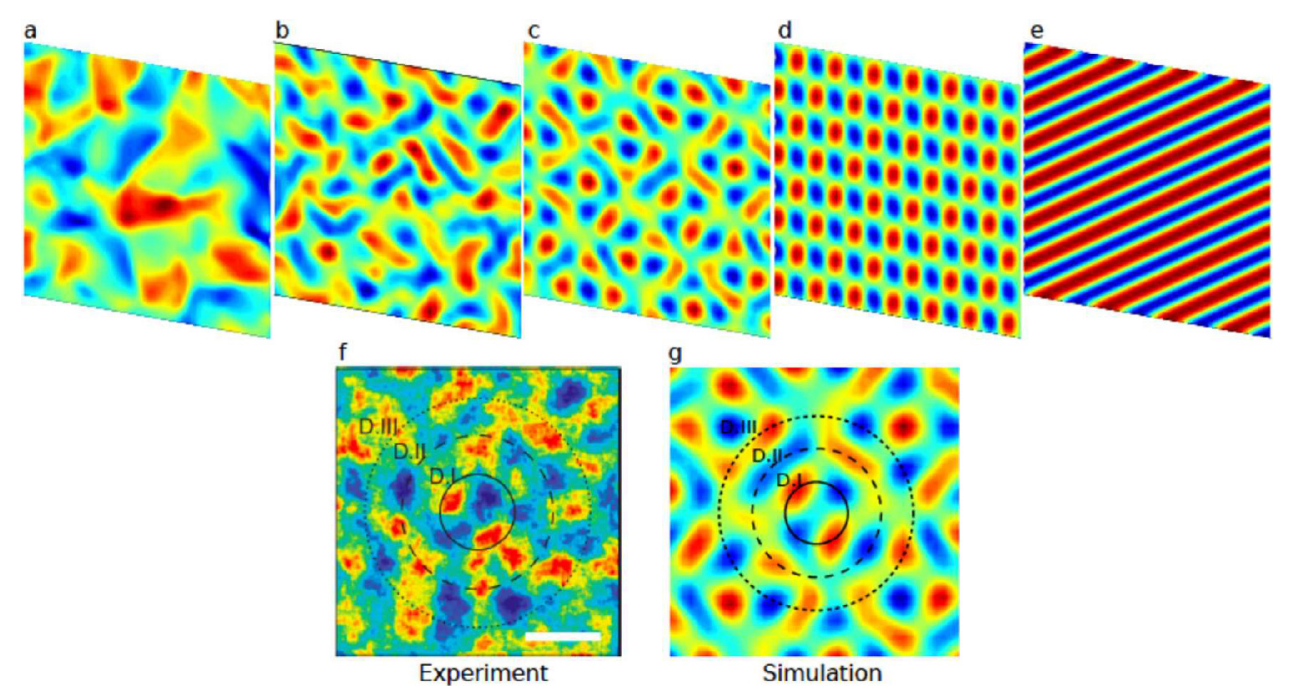

Fig. 4. Emergence of a vortex-lattice in a weakly ordered active nematic with increasing friction. (a-e) The colourmap represents the vorticity field. The friction is increased successively from (a) to (e) [48]. (f) and (g) Comparison of the vortex structure found in the experimental measurements of Rossen et al. [49] on dividing endothelial cells and numerical simulations of contractile active nematics. As in [49], the full (D.I), dashed (D.II), and dotted (D.III) lines correspond to distances one, two, and three cell diameters away from the division site, respectively.

the size of the velocity vortices characterising active turbulence. This is indeed the case, with friction stabilising steady flows in a way analagous to confinement. Continuum simulations have predicted that, for low nematic ordering, a vortex lattice is formed (Figs. 4a-4e) [48]. The velocity vortices self-organise into regular arrays interleaved with an ordered lattice of topological defects. Rossen et al. [49] measured the vorticity field around a dividing cell (by averaging over a large number of cells with division axes rotated to point in the same direction). On average a regular pattern of flow vortices formed around the cell, up to $100 \mu \mathrm{m}$ from the division site. This pattern can be reproduced using a continuum active nematic model for a contractile system with substrate friction. Self-organised vortices have also been observed in sperm cells on a surface [52].

Experiments on the microtubule-motor systems have shown that a variety of different patterned states can be obtained by putting the active layer in contact with a smectic A liquid crystal, where the smectic ordering gives rise to highly anisotropic friction $[53,54]$. For example, focal conics in the smectic effectively result in a soft confinement of the liquid crystal to circular domains and patterns of rotating defects similar to those described in Section 2. Application of a magnetic field aligns the passive liquid crystal into layers, and the active nematic organises into antiparallel flowing bands along the smectic layers. This orientation is because the smectic can flow only within its layers, and hence couples to the active nematic causing dissipation parallel but not perpendicular to the layers.

\section{Discussion}

Controlling active flows provides opportunities for obtaining useful power from active suspensions, driving nanomachines or providing routes to active self assembly. We 
summarise some initial thoughts about how this might be achieved. We concentrate mostly on collective motion of active matter, while noting that a great deal of work has been undertaken on designing single active swimmers and colloids to act as microrobots.

A combination of activity and asymmetric geometries leads to directed motion and the possibility of sorting and transport. For example Galajdi et al. [55] placed bacteria in a chamber containing a line of v-shaped funnels and found that the bacteria preferentially collected on one side of the obstacles, away from the point of the ' $v$ '. Further experiments and theories, reviewed in [56], indicate that the tendency of the bacteria to follow the obstacle walls, together with the activity and asymmetry of the obstacles, are key contributions to the sorting. Similar behaviour has been seen for cells in asymmetric geometries: as the direction and speed of the motion depends on the details of the cell motility this suggests a route for sorting different kinds of cells $[56,57]$. If similar $\mathrm{v}$-shaped obstacles are placed in a bath of bacteria exhibiting active turbulence, and are free to move within the sample chamber, they are transported by the activity. This is due to bacteria that are trapped near the point of the ' $v$ ' and align to push it forwards, while being shielded from the external turbulent flows [58]. Moreover, using passive lyotropic liquid crystals has been shown to provide a means of controlling active matter by directing bacterial motion $[59,60]$.

Results investigating ways to induce propulsion using dense active matter are limited, but Thampi et al. [61] performed simulations of circularly-symmetric rotating discs immersed in an active fluid. They found that, if the rotor dimensions were of order the vortex size, a vortex could form around the rotor. Hence it could undergo persistent rotation with the decorrelation time for a change in the direction of the pinned vortex that increased with increasing rotor radius and decreased with increasing activity. If the rotors were patterned into a square array the confinement between them tended to stabilise any active fluctuations, and neighbouring rotors spun in opposite directions, with an infinitely long persistence time.

Of particular interest very recent experiments have shown how active matter might be used for microfluidic transport. Wu et al. [62] demonstrated that it is possible for a suspension of microtubules and molecular motors to autonomously drive flow through $3 \mathrm{D}$, metre-long channels. For channels with a cross section of aspect ratio $\sim 1$ the flow was coherent for all the channel sizes ( $\mu \mathrm{m}$ to $\mathrm{mm}$ ) considered. Once the channel aspect ratio increased beyond $\sim 3$ the coherent flow was replaced by active turbulence. Given the results described in Section 2.2 this is surprising: in two dimensions there is no coherent flow in channels $>\sim$ the vortex size, whereas in three dimensions there is coherent flow in channels of up to at least $\sim 30$ times the vortex scale. Hence this must be an inherently three dimensional phenomenon. The authors observe that the coherent and turbulent flows are distinguished by the behaviour of the nematic layer that wets the confining surfaces of the channel. When the flow become coherent this layer becomes thicker, and aligns, generating shear that drives the coherent flow.

Applying active matter is a field in its infancy, and translating such simple machines into novel technologies will take ingenuity and effort. It is far from obvious whether individual microswimmers, active colloids or molecular machines such as motor proteins will underpin possible applications, or whether the collective power of dense active matter will prove most useful.

Open Access This is an open access article distributed under the terms of the Creative Commons Attribution License (http://creativecommons.org/licenses/by/4.0), which permits unrestricted use, distribution, and reproduction in any medium, provided the original work is properly cited. 


\section{References}

1. S. Ramaswamy, Annu. Rev. Cond. Mat. Phys. 1, 323 (2010)

2. M.C. Marchetti, J.F. Joanny, S. Ramaswamy, T.B. Liverpool, J. Prost, M. Rao, R.A. Simha, Rev. Mod. Phys. 85, 1143 (2013)

3. D. Needleman, Z. Dogic, Nat. Rev. Mat. 2, 17048 (2017)

4. J. Elgeti, R.G. Winkler, G. Gompper, Rep. Prog. Phys. 78, 056601 (2015)

5. V. Hakim, P. Silberzan, Rep. Prog. Phys. 80, 076601 (2017)

6. T. Sanchez, D.T.N. Chen, S.J. DeCamp, M. Heymann, Z. Dogic, Nature 491, 431 (2012)

7. J.L. Moran, J.D. Posner, Annu. Rev. Fluid Mech. 49, 511 (2017)

8. T.E. Angelini, E. Hannezo, X. Trepat, M. Marquez, J.J. Fredberg, D.A. Weitz, PNAS 108, 4714 (2011)

9. A. Creppy, O. Praud, X. Druart, P.L. Kohnke, F. Plouraboué, Phys. Rev. E 92, 032722 (2015)

10. J. Urzay, A. Doostmohammadi, J.M. Yeomans, J. Fluid Mech. 822, 762 (2017)

11. T.B. Saw, A. Doostmohammadi, V. Nier, L. Kocgozlu, S. Thampi, Y. Toyama, P. Marcq, C.T. Lim, J.M. Yeomans, B. Ladoux, Nature 544, 212 (2017)

12. K. Kawaguchi, R. Kageyama, M. Sano, Nature 545, 327 (2017)

13. Y. Hatwalne, S. Ramaswamy, M. Rao, R.A. Simha, Phys. Rev. Lett. 92, 118101 (2004)

14. S.P. Thampi, J.M. Yeomans, Eur. Phys. J. Special Topics 225, 651 (2016)

15. H. Wioland, F.G. Woodhouse, J. Dunkel, J.O. Kessler, R.E. Goldstein, Phys. Rev. Lett. 110, $268102(2013)$

16. E. Lushi, H. Wioland, R.E. Goldstein, PNAS 111, 9733 (2014)

17. H. Wioland, F.G. Woodhouse, J. Dunkel, R.E. Goldstein, Nat. Phys. 12, 341 (2016)

18. T. Gao, M.D. Betterton, A.S. Jhang, M.J. Shelley, Phys. Rev. Fluids 2, 093302 (2017)

19. K. Doxzen, S.K. Vedula, M.C. Leong, H. Hirata, N.S. Gov, A.J. Kabla, B. Ladoux, C.T. Lim, Integr. Biol. 5, 1026 (2013)

20. T.N. Shendruk, A. Doostmohammadi, K. Thijssen, J.M. Yeomans, Soft Matter 13, 3853 (2017)

21. F.G. Woodhouse, R.E. Goldstein, Phys. Rev. Lett. 109, 168105 (2012)

22. M. Ravnik, J.M. Yeomans, Phys. Rev. Lett. 110, 026001 (2013)

23. M. Neef, K. Kruse, Phys. Rev. E 90, 052703 (2014)

24. M.M. Norton, A. Baskaran, A. Opathalage, B. Langeslay, S. Fraden, A. Baskaran, M. Hagan, Phys. Rev. E 97, 012702 (2018)

25. P. Guillamat, J. Ignés-Mullol, F. Sagués, Nat. Commun. 8, 564 (2017)

26. M. Theillard, R. Alonso-Matilla, D. Saintillan, Soft Matter 13, 363 (2017)

27. M. Deforet, V. Hakim, H.G. Yevick, G. Duclos, P. Silberzan, Nat. Commun. 5, 3747 (2014)

28. J. Notbohm, S. Banerjee, K.J.C. Utuje, B. Gweon, H. Jang, Y. Park, J. Shin, J.P. Butler, J.J. Fredberg, M.C. Marchetti, Biophys. J. 110, 2729 (2016)

29. A. Doostmohammadi, S.P. Thampi, T.B. Saw, C.T. Lim, B. Ladoux, J.M. Yeomans, Soft Matter 11, 7328 (2015)

30. M.J. Siedlik, S. Manivannan, I.G. Kevrekidis, C.M. Nelson, Biophys. J. 112, 2419 (2017)

31. R. Voituriez, J.F. Joanny, J. Prost, Europhys. Lett. 70, 404 (2005)

32. D. Marenduzzo, E. Orlandini, M.E. Cates, J.M. Yeomans, Phys. Rev. E 76, 031921 (2007)

33. S. Edwards, J.M. Yeomans, Europhys. Lett. 85, 18008 (2009)

34. R.A. Simha, S. Ramaswamy, Phys. Rev. Lett. 89, 058101 (2002)

35. R. Green, J. Toner, V. Vitelli, Phys. Rev. Fluids 2, 104201 (2017)

36. A. Doostmohammadi, T.N. Shendruk, K. Thijssen, J.M. Yeomans, Nat. Commun. 8, 15326 (2017)

37. R. Ramaswamy, F. Jülicher, Sci. Rep. 6, 20838 (2016)

38. M. Sano, K. Tamai, Nat. Phys. 12, 249 (2016)

39. G. Lemoult, L. Shi, K. Avila, S.V. Jalikop, M. Avila, B. Hof, Nat. Phys. 12, 254 (2016)

40. E.J. Hemingway, P. Mishra, M.C. Marchetti, S.M. Fielding, Soft Matter 12, 7943 (2016)

41. H. Wioland, E. Lushi, R.E. Goldstein, New J. Phys. 18, 075002 (2016) 
42. S.K. Vedula, M.C. Leong, T.L. Lai, P. Hersen, A.J. Kabla, C.T. Lim, B. Ladoux, PNAS 109, $12974(2012)$

43. A.K. Marel, M. Zorn, C. Klingner, R. Wedlich-Söldner, E. Frey, J.O. Rädler, Biophys. J. 107, 1054 (2014)

44. A.K. Marel, N. Podewitz, M. Zorn, J.O. Rädler, J. Elgeti, New J. Phys. 16, 115005 (2014)

45. V. Nier, M. Deforet, G. Duclos, H.G. Yevick, O. Cochet-Escartin, P. Marcq, P. Silberzan, PNAS 112, 9546 (2015)

46. V. Tarle, E. Gauquelin, S.R.K. Vedula, J. D'Alessandro, C.T. Lim, B. Ladoux, N.S. Gov, Phys. Biol. 14, 035001 (2017)

47. F.G. Woodhouse, R.E. Goldstein, PNAS 110, 14132 (2013)

48. A. Doostmohammadi, M.F. Adamer, S.P. Thampi, J.M. Yeomans, Nat. Commun. 7, 10557 (2016)

49. N.S. Rossen, J.M. Tarp, J. Mathiesen, M.H. Jensen, L.B. Oddershede, Nat. Commun. $\mathbf{5}, 5720(2014)$

50. S.P. Thampi, R. Golestanian, J.M. Yeomans, Phys. Rev. E 90, 062307 (2014)

51. P. Guillamat, J. Ignés-Mullol, F. Sagués, Phys. Rev. E 94, 060602 (2016)

52. I.H. Riedel, K. Kruse, J. Howard, Science 309, 300 (2005)

53. P. Guillamat, J. Ignés-Mullol, F. Sagués, PNAS 113, 5498 (2016)

54. P. Guillamat, J. Ignés-Mullol, F. Sagués, Mol. Crys. Liq. Cryst. 646, 226 (2017)

55. P. Galajda, J. Keymer, P. Chaikin, R. Austin, J. Bacteriol. 189, 8704 (2007)

56. C.J. Olson Reichhardt, C. Reichhardt, Annu. Rev. Condens. Matter Phys 8, 51 (2017)

57. G. Mahmud, C.J. Campbell, K.J.M. Bishop, Y.A. Komarova, O. Chaga, S. Soh, S. Huda, K. Kandere-Grzybowska, B.A. Grzybowski, Nat. Phys. 5, 606 (2009)

58. A. Kaiser, A. Peshkov, A. Sokolov, B. ten Hagen, H. Lowen, I.S. Aranson, Phys. Rev. Lett. 112, $158101(2014)$

59. S. Zhou, A. Sokolov, O.D. Lavrentovich, I.S. Aranson, PNAS 111, 1265 (2014)

60. C. Peng, T. Turiv, Y. Guo, Q-H. Wei, O.D. Lavrentovich, Science 354, 882 (2016)

61. S.P. Thampi, A. Doostmohammadi, T.N. Shendruk, R. Golestanian, J.M. Yeomans, Sci. Adv. 2, e1501854 (2016)

62. K.T. Wu, J.B. Hishamunda, D.T.N. Chen, S.J. DeCamp, Y.W. Chang, A. FernándezNieves, S. Fraden, Z. Dogic, Science 355, 1979 (2017) 